\title{
Near-infrared intraoperative imaging for minimally invasive pulmonary metastasectomy for sarcomas
}

Jarrod D. Predina, MD, MTR, ${ }^{\mathrm{a}, \mathrm{b}}$ Andrew D. Newton, MD, ${ }^{\mathrm{a}, \mathrm{c}}$ Christopher Corbett, BA, ${ }^{\mathrm{a}, \mathrm{b}}$ Michael Shin, BA, ${ }^{\mathrm{a}, \mathrm{b}}$ Lydia Frenzel Sulfyok, AB, ${ }^{\mathrm{a}, \mathrm{b}}$ Olugbenga T. Okusanya, MD, Edward J. Delikatny, PhD, ${ }^{a}$ Shuming Nie, PhD, ${ }^{d}$ Colleen Gaughan, MD, ${ }^{a, b}$ Doraid Jarrar, MD, Taine Pechet, MD, a,b John C. Kucharczuk, MD, ${ }^{a, b}$ and Sunil Singhal, MD ${ }^{a, b}$

\section{ABSTRACT}

Background: Complete pulmonary metastasectomy for sarcoma metastases provides patients an opportunity for long-term survival and possible cure. Intraoperative localization of preoperatively identified metastases and identification of occult lesions can be challenging. In this trial, we evaluated the efficacy of near-infrared (NIR) intraoperative imaging using second window indocyanine green during metastasectomy to identify known metastases and to detect occult nodules.

Methods: Thirty patients with pulmonary nodules suspicious for sarcoma metastases were enrolled in an open-label, feasibility study (NCT02280954). All patients received intravenous indocyanine green $(5 \mathrm{mg} / \mathrm{kg}) 24$ hours before metastasectomy. Patients 1 through 10 (cohort 1) underwent metastasectomy via thoracotomy to assess fluorescence patterns of nodules detected by traditional methods (preoperative imaging and intraoperative visualization/bimanual palpation). After confirming reliability within cohort 1 , patients 11 through 30 (cohort 2) underwent video-assisted thoracic surgery metastasectomy with NIR imaging.

Results: In cohort 1, 14 out of 16 preoperatively identified pulmonary metastases $(87.5 \%)$ displayed tumor fluorescence. Nonfluorescent metastases were deeper than fluorescent metastases $(2.1 \mathrm{~cm}$ vs $1.3 \mathrm{~cm} ; P=.03)$. Five out of 5 metastases identified during thoracotomy displayed fluorescence. NIR imaging identified 3 additional occult lesions in this cohort. In cohort 2, 33 out of 37 known pulmonary metastases $(89.1 \%)$ displayed fluorescence. Nonfluorescent tumors were deeper than $2.0 \mathrm{~cm}(P=.007)$. NIR imaging identified 24 additional occult lesions. Of 24 occult lesions, $21(87.5 \%)$ were confirmed metastases and the remaining 3 nodules were lymphoid aggregates.

Conclusions: NIR intraoperative imaging with indocyanine green $(5 \mathrm{mg} / \mathrm{kg}$ and 24 hours before surgery) localizes known sarcoma pulmonary metastases and identifies otherwise occult lesions. This approach may be a useful intraoperative adjunct to improve metastasectomy. (J Thorac Cardiovasc Surg 2019;157:2061-9)

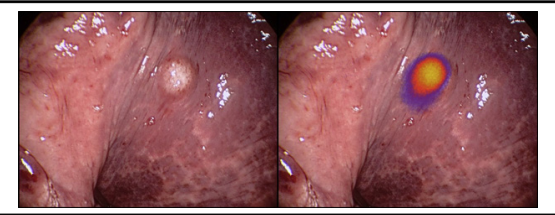

Near-infrared intraoperative imaging of sarcoma metastasis during VATS metastasectomy.

Central Message

Near-infrared intraoperative imaging with second window indocyanine green may be a useful tool that can improve identification of sarcoma metastases to the lung.

\section{Perspective}

During pulmonary resection for sarcoma metastases, intraoperative localization of preoperatively identified lesions and identification of occult metastases can be challenging. In this trial, we demonstrate that near-infrared intraoperative imaging with second window indocyanine green improves identification of known metastases and detection of occult nodules during metastasectomy.

See Commentaries on pages 2070 and 2072.
From the ${ }^{\mathrm{a} C e n t e r}$ for Precision Surgery, ${ }^{\mathrm{b}}$ Division of Thoracic Surgery, Department of Surgery, and ${ }^{\mathrm{c}}$ Department of Surgery, Perelman School of Medicine at the University of Pennsylvania, Philadelphia, $\mathrm{Pa}$; and ${ }^{\mathrm{d}}$ Department of Chemistry, University of Illinois, Champaign, Ill.

Dr Predina was supported by a grant from the American Philosophical Society, by the National Institutes of Health (grant No. F32 CA21040), and by the Association for Academic Surgery. Dr Singhal was supported by the National Institutes of Health (grant No. R01 CA193556).

Read at the 98th Annual Meeting of The American Association for Thoracic Surgery, San Diego, California, April 28-May 1, 2018.
Received for publication May 23, 2018; revisions received Oct 18, 2018; accepted for publication Oct 31, 2018.

Address for reprints: Sunil Singhal, MD, Center for Precision Surgery, Perelman School of Medicine at the University of Pennsylvania, 6 White Bldg, 3400 Spruce St, Philadelphia, PA 19104 (E-mail: Sunil.singhal@uphs.upenn.edu). 0022-5223/\$36.00

Copyright (C) 2018 by The American Association for Thoracic Surgery https://doi.org/10.1016/j.jtcvs.2018.10.169 


\section{Abbreviations and Acronyms}

$\mathrm{EPR}=$ enhanced permeability and retention effect

ICG = indocyanine green

NIR $=$ near infrared

TBR $=$ tumor-to-background fluorescent ratio

VATS $=$ video-assisted thoracoscopic surgery

\section{Scanning this QR code will}

take you to the article title page to access supplementary information. To view the AATS Annual Meeting Webcast, see the URL next to the webcast thumbnail.

Sarcomas represent a heterogenous group of neoplasms arising from mesenchymal cell origins and account for 15,000 new cancer cases each year. ${ }^{1}$ Isolated pulmonary metastases are observed in approximately $20 \%$ of patients with soft tissue sarcoma ${ }^{2}$ and approximately $40 \%$ of patients with bone sarcoma. ${ }^{3}$ In these circumstances, pulmonary metastasectomy has become a well-accepted intervention that seems to provide the only opportunity to improve long-term survivorship. ${ }^{2-6}$

During metastasectomy, the goal is complete resection of disease. Unfortunately, intraoperative identification of small lesions can be challenging, particularly in the scenario of soft tissue sarcomas, which are soft and therefore challenging to identify by palpation. In addition to localizing known lesions, surgeons must also identify small lesions that may be missed by preoperative imaging. To accomplish this task, a number of studies suggest metastasectomy via thoracotomy as an optimal approach as it allows for bimanual lung palpation. ${ }^{7-9}$ Indeed, multiple reports have revealed an increase in the number of resected nodules during thoracotomy compared with video-assisted thoracoscopic surgery (VATS), ${ }^{9}$ with occult lesion detection rates as high as $35 \%{ }^{7,8}$ Although open approaches offer improved visualization and palpation compared with VATS, thoracotomy carries higher morbidity than minimally invasive approaches, and it may complicate future procedures.

A best-of-both-worlds scenario incorporates a reliable, minimally invasive technique that allows for reproducible detection of both known and radiographically occult lesions. One potential approach is intraoperative imaging, also referred to fluorescence-guided surgery, a technique that incorporates systemic delivery of near-infrared (NIR) contrast agents that preferentially accumulate in cancer deposits and result in tumor fluorescence during resection. Our group has previously utilized intraoperative imaging for localization of several common thoracic malignancies including non-small cell lung cancer, ${ }^{10}$ mesothelioma, ${ }^{11}$ anterior mediastinal neoplasms, ${ }^{12}$ and nonsarcoma pulmonary metastases. ${ }^{13}$ Our primary approach has involved systemic delivery of the NIR contrast agent, indocyanine green (ICG), with fluorescence-guided surgery occurring 24 hours after drug delivery (during the so-called second window). ICG is an Food and Drug Administrationapproved NIR optical contrast that has the propensity to accumulate in a diverse array of tumor histologies via the enhanced permeability and retention effect (EPR). ${ }^{10,13}$ In addition to generalizability, ICG is relatively inexpensive and has an excellent safety profile.

Given previous successes involving NIR intraoperative imaging with ICG, we designed a clinical trial to determine whether this approach could reliably identify known sarcoma metastases and improve detection radiographically occult metastases during minimally invasive pulmonary metastasectomy. In this report, we describe our experiences involving 30 patients who presented for pulmonary metastasectomy for sarcomas. Within the first 10 patients (cohort 1), we confirm the ability of NIR imaging to identify lesions discovered by preoperative

TABLE 1. Patient characteristics

\begin{tabular}{|c|c|c|c|}
\hline \multicolumn{2}{|c|}{$\begin{array}{c}\text { Cohort } 1 \text { ( } \mathrm{n}=10 \text { patients, } \\
\mathrm{n}=16 \text { lesions })\end{array}$} & \multicolumn{2}{|c|}{$\begin{array}{c}\text { Cohort } 2(n=20 \text { patients, } \\
n=37 \text { lesions })\end{array}$} \\
\hline Variable & $\mathbf{N}$ & Variable & $\mathbf{N}$ \\
\hline Age (y) & & Age (y) & \\
\hline$<40$ & 3 & $<40$ & 6 \\
\hline$\geq 41-<60$ & 3 & $\geq 41-<60$ & 11 \\
\hline$\geq 60$ & 4 & $\geq 60$ & 3 \\
\hline Mets by $\mathrm{CT}^{*}$ & & Mets by $\mathrm{CT}^{*}$ & \\
\hline 1 & 7 & 1 & 12 \\
\hline 2 & 1 & 2 & 3 \\
\hline 3 & 1 & 3 & 2 \\
\hline 4 & 1 & 4 & 2 \\
\hline & & 5 & 1 \\
\hline Metastasis size $(\mathrm{cm})$ & & Metastasis size $(\mathrm{cm})$ & \\
\hline$<1$ & 6 & $<1$ & 19 \\
\hline$\geq 1-<2$ & 6 & $\geq 1-<2$ & 3 \\
\hline$\geq 2$ & 4 & $\geq 2$ & 5 \\
\hline Tumor histology & & umor histology & \\
\hline Leiomyosarcoma & 3 & MFH & 4 \\
\hline Ewing's Sarcoma & 2 & Osteosarcoma & 4 \\
\hline Osteosarcoma & 2 & GIST & 2 \\
\hline Other & 3 & Ewing's sarcoma & 2 \\
\hline & & Leiomyosarcoma & 2 \\
\hline & & Rhabdosarcoma & 2 \\
\hline & & Other & 4 \\
\hline
\end{tabular}

CT, Computed tomography; Mets, metastastes; $M F H$, malignant fibrous histiocytoma; GIST, gastrointestinal stromal tumor. *Number of pulmonary Mets is based only on preoperative imaging and includes only lesions within the hemithorax undergoing resection. 
imaging and bimanual palpation via thoracotomy. After confirming reliability of NIR imaging during thoracotomy, we validated this approach during minimally invasive metastasectomy in 20 patients (cohort 2).

\section{MATERIALS AND METHODS Study Drug}

Patients received a 5-mg/kg infusion of ICG (Akorn Pharmaceuticals, Decatur, Ga) 24 hours before resection. ICG is an NIR optical agent with an excitation wavelength of $805 \mathrm{~nm}$ and an emission wavelength of $830 \mathrm{~nm}$. In situ, real-time fluorescent imaging was performed using the Iridium system optimized for detection of ICG (Visionsense Corp,
Philadelphia, Pa) as previously described by our group. ${ }^{14}$ The Iridium is a high-definition, dual band (white light and NIR) camera system capable of emitting and detection light in the NIR spectrum. During both open and minimally invasive resections, NIR imaging was obtained using a 5-mm, $0^{\circ}$ thoracoscope.

\section{Study Design}

This nonrandomized, open-label study (ClinicalTrials.gov: NCT02280954) was approved by the University of Pennsylvania Institutional Review Board. Thirty patients provided informed consent and were enrolled in 2 cohorts between November 2014 and September 2017. The primary goals were to determine whether NIR imaging after systemic ICG delivery could reliably identify occult sarcoma metastases.
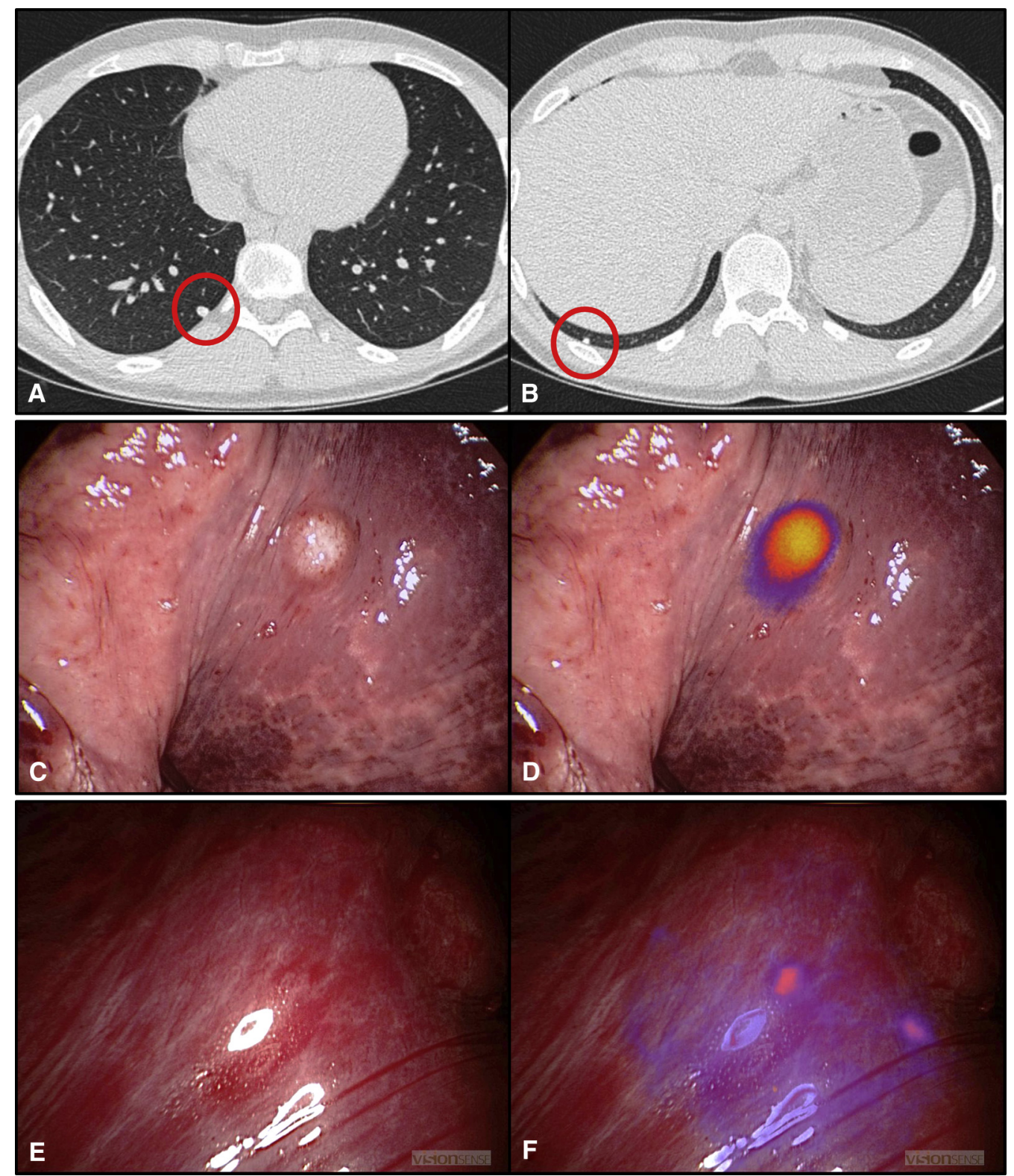

FIGURE 1. Representative example of near infrared (NIR) intraoperative imaging during thoracotomy for subject with osteosarcoma metastases. A and B, Preoperative high-resolution computed tomography revealing multiple right-sided pulmonary metastases (red circles). C, Traditional brightfield view of known basilar metastasis. D, NIR merged view of known basilar metastasis. E, Bright field view of right middle lobe revealing no obvious disease. F, NIR merged view demonstrating several occult metastases measuring 3 to $5 \mathrm{~mm}$. 
Cohort 1 was designed for proof-of-concept. Ten patients with a history of sarcoma and suspicious pulmonary nodules on axial imaging were enrolled. Each patient underwent unilateral thoracotomy during which the surgeon identified preoperatively identified lesions then assessed the remaining lung parenchyma for additional lesions by both visualization and bimanual palpation. All identified lesions were then assessed by NIR imaging to determine fluorescence. Finally, the hemithorax was re-evaluated with NIR imaging to determine whether additional lesions were identifiable by fluorescence alone. Each identified lesion was wedge resected and reviewed by a pathologist.

Cohort 2 was a validation cohort. Twenty patients with pulmonary lesions suspicious for sarcoma metastases were infused with ICG, then underwent VATS metastasectomy. After localizing known lesions and identifying additional lesions by visualization and tactile feedback (ie, finger palpation), NIR imaging was utilized to confirm fluorescence. After confirming fluorescence of lesions identified by traditional means, the hemithorax was examined by NIR imaging to identify additional lesions. Again, all identified lesions were wedge resected and submitted for pathologic review.

\section{Statistical Analysis}

Data are presented as mean \pm standard deviation unless otherwise noted. Given the small sample size, differences between 2 groups were compared by the Mann-Whitney test. All comparisons were made using Stata Statistical Software release 14 (StataCorp, College Station, Tex).

\section{RESULTS \\ Patient Data}

Between November 2014 and September 2017, 30 patients ( 16 men) with a mean age of 51.5 years (range, 23-79 years) were enrolled after meeting inclusion criteria. Among 30 enrolled patients, a total of 53 suspicious lesions (16 in cohort 1 and 37 in cohort 2) were identified by preoperative imaging. Nineteen patients presented with a single suspicious lesion, 4 presented with 2 lesions, 3 presented with 3 lesions, 3 presented with 4 lesions, and 1 patient presented with 5 lesions. The most common tumor histologies included osteosarcoma $(\mathrm{n}=6)$, leiomyosarcoma $(\mathrm{n}=5)$, malignant fibrous histiocytoma $(\mathrm{n}=4)$, Ewing's sarcoma $(\mathrm{n}=4)$, rhabdosarcoma $(\mathrm{n}=2)$, and liposarcoma $(\mathrm{n}=2)$ and gastrointestinal stromal tumor $(\mathrm{n}=2)$. The mean size of preoperatively identified lesions was $1.6 \pm 0.54 \mathrm{~cm}$ (range, $0.5-3.2 \mathrm{~cm}$ ). All patients received the full ICG infusion without toxicity. A summary of patient characteristics per enrollment cohort is described in Table 1.

Cohort 1: Metastases identified by preoperative imaging and intraoperative visualization/bimanual palpation display fluorescence. Among the 10 patients enrolled in cohort 1, 16 suspicious lesions were identified by preoperative imaging. During thoracotomy, each lesion was identified by visualization or bimanual palpation. Of these 16 known lesions, $14(87.5 \%)$ displayed tumor fluorescence upon NIR inspection (mean tumor-tobackground fluorescent ratio [TBR], 3.1; range, 2.7-4.2). The nonfluorescent metastases were deeper than fluorescent metastases $(2.1 \mathrm{~cm}$ vs $1.3 \mathrm{~cm} ; P=.03)$. Both nodules displayed signal upon tumor bisection on the back table.

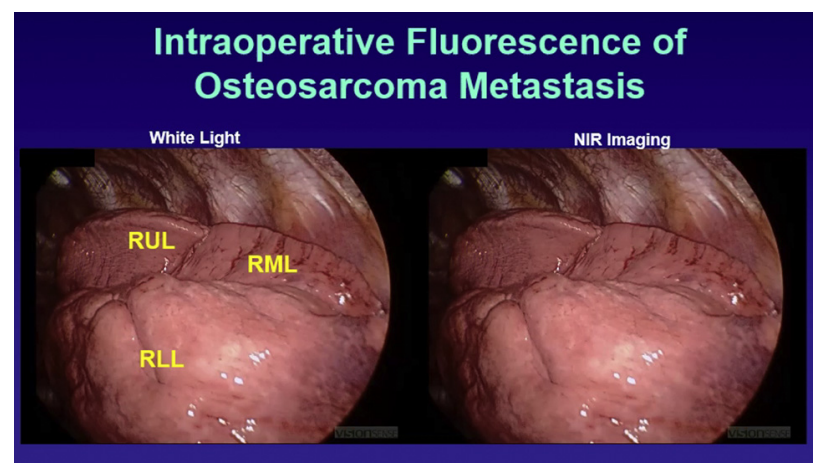

VIDEO 1. Intraoperative near-infrared imaging of a patient with history of osteosarcoma undergoing pulmonary metastasectomy. Video available at: https://www.jtcvs.org/article/S0022-5223(18)33286-0/fulltext.

In addition to the 16 lesions identified by imaging, 5 metastases were identified by palpation/visualization. Each of these lesions also displayed fluorescence with a mean TBR of 3.3 (range, 2.8-4.1). Finally, NIR imaging identified 3 additional metastases (range, $3-5 \mathrm{~mm}$ ) that were otherwise unidentifiable. Those lesions identified intraoperatively by NIR alone trended to be smaller than those identified intraoperatively by bimanual palpation (4.2 $\mathrm{mm}$ vs $5.6 \mathrm{~mm} ; P=.08$ ) A representative example is provided in Figure 1 and Video 1. Cohort 1 data are summarized in Figure 2.

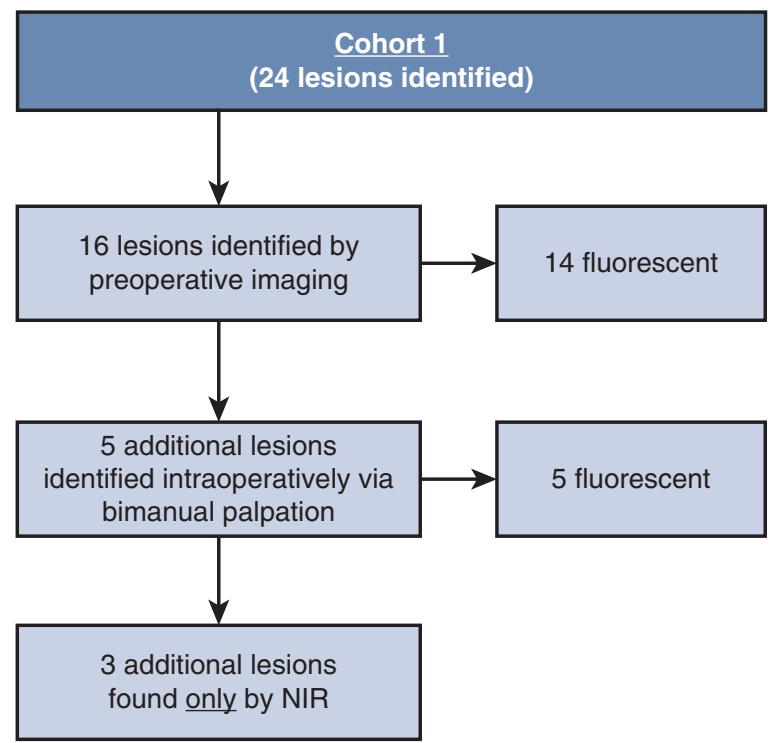

FIGURE 2. Summary of metastases identified within cohort 1 . Within the 10 patients enrolled in cohort 1 , a total 24 metastases were identified using a combination of preoperative computed tomography (CT), intraoperative palpation, and near infrared (NIR) imaging. Sixteen lesions were identified by preoperative $\mathrm{CT}$, with 14 being fluorescent. Five lesions were identified by bimanual palpation, and each was fluorescent. Three additional lesions were identified by NIR imaging alone. 

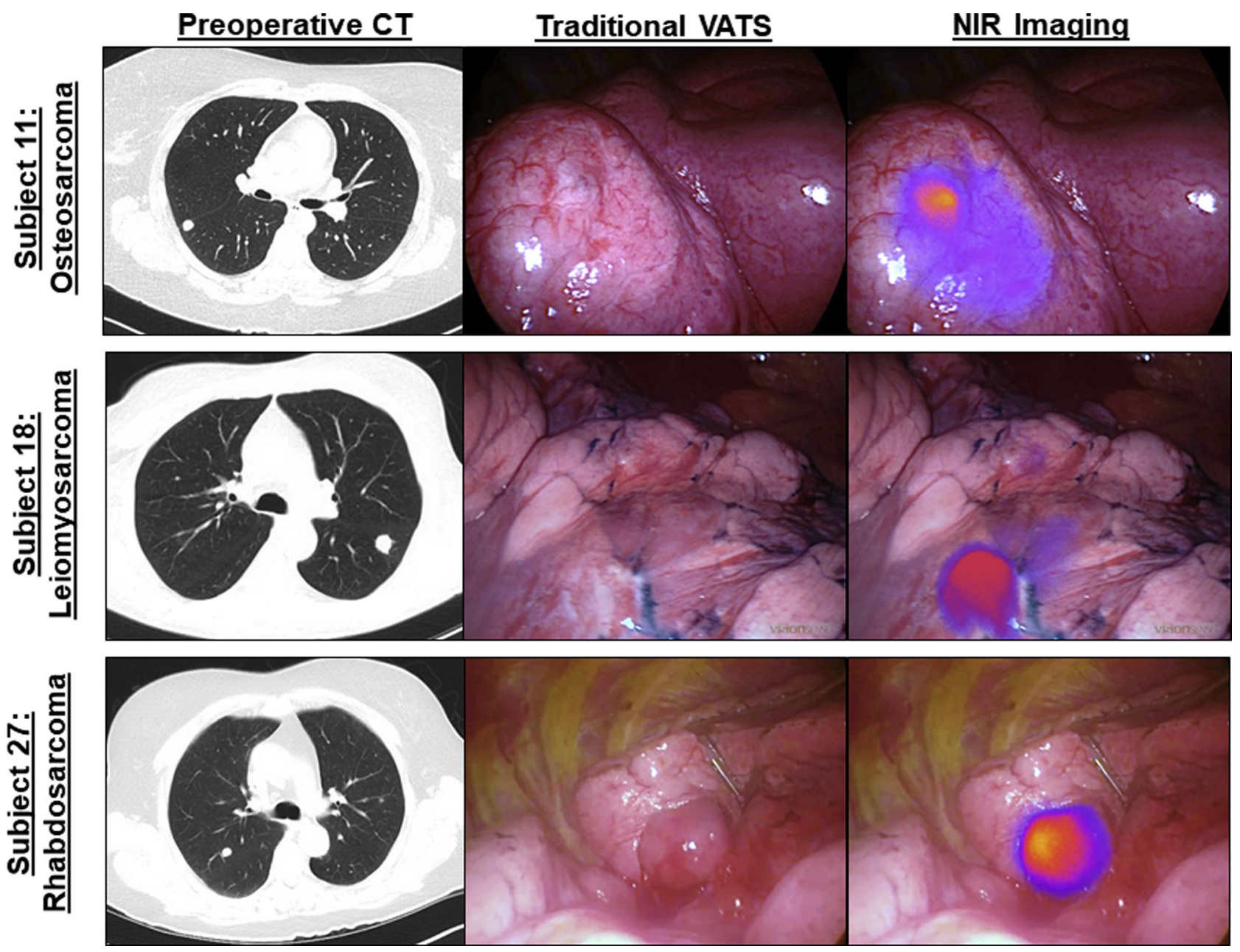

FIGURE 3. Representative examples of near-infrared (NIR) intraoperative imaging during video-assisted thoracoscopic surgery (VATS) metastasectomy. Left column, Preoperative high-resolution computed tomography $(C T)$. Middle column, Bright field (traditional views). Right column, NIR merged view.

Cohort 2: NIR imaging during VATS allows detection of additional pulmonary metastases. Within cohort 2, 33 out of 37 preoperatively identified pulmonary metastases $(89.1 \%$ ) displayed fluorescence (mean TBR, $2.9 \pm 0.3$ ). Representative examples are provided in Figure 3. All nonfluorescent tumors were deeper than $2.0 \mathrm{~cm}(P=.007)$. NIR imaging identified 24 additional fluorescent lesions during VATS, with a mean TBR of $2.8 \pm 2.6-3.0$ and a mean size of $4 \mathrm{~mm}$ (range, 2-6 mm). Of the 24 occult lesions, $21(87.5 \%)$ were confirmed metastases and 3 were benign lymphoid aggregates. Cohort 2 data are summarized in Figure 4. Based on these data, NIR imaging was more sensitive than traditional lesion detection approaches $(93.1 \%$ vs $63.8 \% ; P<.05)$. Estimates of specificity, positive-predictive value, and negativepredictive value were statistically similar or could not be reliably compared based on sample data (complete data provided in Table 2).

\section{Feasibility and Clinical Utility}

During NIR imaging, modified thoracoscopes were utilized and high-definition fluorescent videos were displayed in real time using standard operating room monitors. Cost of NIR imaging ranged between $\$ 1500$ and $\$ 2500$ per patient and was primarily driven by cost of ICG. Upon subgroup analysis, we noted that NIR identified more lesions during VATS than during thoracotomy ( $2.6 \mathrm{vs}$ 1.5 lesions per subject; $P=.06$ ). We saw no difference in fluorescence patterns of soft tissue sarcomas (40 of 44 lesions fluoresced) and bone sarcomas (36 of 40 fluoresced) $(P>.05)$. As noted previously, nonfluorescent lesions were deeper than $2.0 \mathrm{~cm}$.

\section{DISCUSSION}

During pulmonary metastasectomy for patients with sarcomas, complete resection is key to improving long-term outcomes. Localization of small pulmonary 


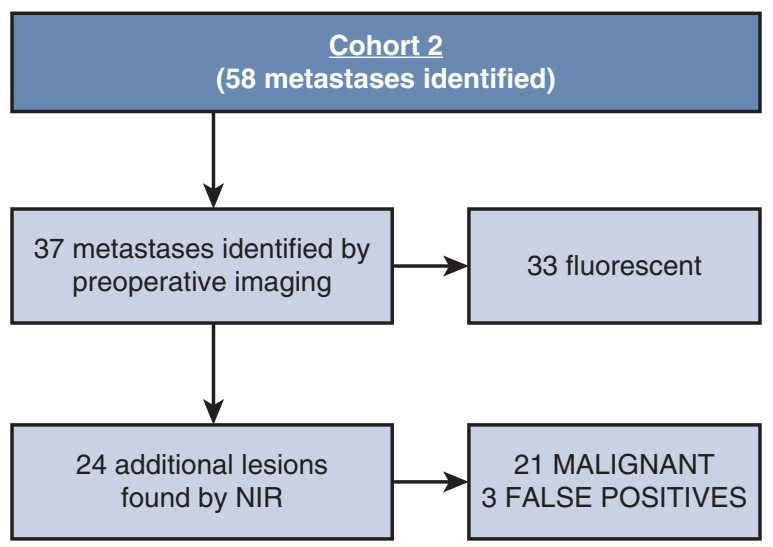

FIGURE 4. Summary of metastases identified within cohort 2 . Among the 20 patients enrolled in cohort 2, 58 metastases were identified using a combination of preoperative computed tomography (CT) and intraoperative near-infrared (NIR) imaging. Thirty-three of 37 lesions identified by preoperative CT were fluorescent. NIR imaging identified 24 additional lesions during video-assisted thoracic surgery, 21 were histologically confirmed metastases.

nodules and identifying occult lesions is a clinical challenge, and we present data suggesting that NIR intraoperative imaging may be an effective approach to assist thoracic surgeons. We demonstrate that NIR imaging by infusing high-dose ICG and then imaging occurring 24 hours later (an approach we have previously called Tumor Glow) can identify metastases that usually require bimanual palpation during thoracotomy. We also show data suggesting that this approach can identify otherwise occult lesions during open and minimally invasive metastasectomy. Tumor fluorescence was independent of sarcoma histology; however, it is best suited for lesions within $2 \mathrm{~cm}$ of the pleural surface.
ICG has traditionally been to assess perfusion of tissues $^{15-17}$; however, we have modified delivery parameters such that ICG may be deployed as a tumor mapping agent. ${ }^{18}$ To accomplish this objective, we intravenously deliver ICG at a much higher dose $(5 \mathrm{mg} / \mathrm{kg}$ ) and image at a later time (24 hours after delivery, during the so-called second window). We call this imaging approach Tumor Glow. Feasibility of NIR imaging in the second window is feasible due to dye accumulation in tissues with abnormally leaky capillaries and/or increased pressure gradients through the EPR effect. Although we have found successes using ICG as a tumor mapping agent for other solid tumor histologies, ${ }^{10-13}$ our experiences involving sarcomatous pulmonary metastases are among the most encouraging. We believe that excellent reproducibility and high TBRs observed are a result of low amounts of dye accumulation in benign parenchyma, which results in high NIR signal within tumors relative to background. In other solid tumors, such as non-small cell lung cancer or mesothelioma, tumors accumulate dye similar to sarcoma metastases; however, surrounding tissues are typically chronically inflamed or dysplastic and therefore exhibit EPR, which promotes off-target dye accumulation. In addition to physiologic considerations, the peripheral anatomic location of metastases is favorable to NIR imaging approaches. ${ }^{19}$

In this study, we found that NIR imaging was capable of localizing those metastases identified by bimanual during thoracotomy. NIR imaging also identified occult lesions in patients undergoing open and minimally invasive metastasectomy, with more lesions per patient being observed during VATS. The latter finding was not surprising given the inability for surgeons to perform thorough

TABLE 2. Test characteristics of traditional intraoperative metastasis detection* versus near infrared (NIR) intraoperative imaging $\dagger$ during video-assisted thoracic surgery (VATS) metastasectomy (based on 61 identified lesions)

\begin{tabular}{|c|c|c|}
\hline & Sarcoma metastasis $\ddagger$ & Benign lesion \\
\hline \multicolumn{3}{|c|}{ Traditional intraoperative detection } \\
\hline Identified* & 37 & 0 \\
\hline \multirow[t]{2}{*}{ Occult $\dagger$} & 21 & 3 \\
\hline & $\begin{array}{l}\text { Sensitivity: } 63.8 \%(95 \% \text { CI, } 50.1 \%-76.0 \%) \\
\text { Specificity: } 100 \%(95 \% \text { CI, } 29.2 \%-100 \%) \\
\text { PPV: } 100 \% \\
\text { NPV: } 12.5 \%(96 \% \text { CI, } 9.2 \%-16.7 \%)\end{array}$ & \\
\hline \multicolumn{3}{|l|}{ NIR intraoperative imaging } \\
\hline Fluorescent (TBR $\geq 2$ ) & 54 & 3 \\
\hline \multirow[t]{2}{*}{ Nonfluorescent $(\mathrm{TBR}<2)$} & 4 & 0 \\
\hline & $\begin{array}{l}\text { Sensitivity: } 93.1 \%(95 \% \text { CI, } 83.3 \%-98.1 \%) \\
\text { Specificity: } 0 \%(95 \% \text { CI, 0\%-70.6\%) } \\
\text { PPV: } 94.7 \%(95 \% \text { CI, } 94.3 \%-95.1 \%) \\
\text { NPV: } 0 \%\end{array}$ & \\
\hline
\end{tabular}

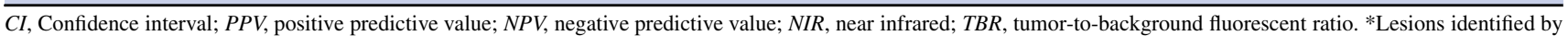
traditional detection included those lesions detected by preoperative imaging, intraoperative visualization, or finger palpation. $\dagger$ If a lesion was initially identified by NIR imaging, it was considered occult even if retrospectively identified by CT or palpation. ‡Pathology considered the gold standard. 
bimanual inspection during VATS. Interestingly, several of the lesions identified by VATS NIR imaging (cohort 2) were palpable on back table evaluation, which suggests that they may have been identified via thoracotomy. These data suggest that this technology may be a useful adjunct in those patients undergoing thoracoscopic resections.

We believe identification of additional metastases by NIR of imaging is of clinical relevance. Although one would hope that identification of additional metastases would directly correlate to improved long-term survival, we recognize that this may not be the case because NIR imaging cannot routinely identify lesions smaller than $5 \mathrm{~mm}$ and those situated deeper than $2 \mathrm{~cm}$. Despite these limitations, identification of occult lesions may prove beneficial to patients by increasing disease free intervals and limiting the need for additional interventions. More immediately, we did observe that identification of occult lesions influenced postoperative follow-up and adjuvant therapy. To elaborate, we identified a subgroup of patients who were scheduled for metastasectomy of isolated lesion; however, were found to have multiple additional lesions upon NIR imaging. Because of these findings, these patients were scheduled to more aggressive adjuvant protocols and more frequent surveillance imaging. Despite these observations, we acknowledge that additional follow-up data and larger studies are needed to fully understand how NIR findings influence postoperative outcomes and whether those benefits outweigh morbidity associated with downstream interventions.

NIR imaging with ICG does have several appreciable limitations. As alluded to above, current NIR imaging devices can only detect lesions within $2 \mathrm{~cm}$ of the pleural surface. Although most metastases are peripheral, there are certainly those that reside deeper in the lung. In addition to deeper lesions, NIR imaging can only reproducibly identify lesions larger than $5 \mathrm{~mm}$. Lesions smaller than this are not always identifiable, particularly when deeply located. Finally, the cost of this technology is currently approximately $\$ 1500$ to $\$ 2500$ per patient. In efforts to improve cost-effectiveness, we are conducting a dose de-escalation trial and are optimistic that costs can be reduced by $60 \%$ (data not published).

Notwithstanding these limitations, we feel that NIR imaging with ICG can be a valuable tool to improve management of patients with sarcomatous pulmonary metastases. Future studies will primarily involve modifications to drug delivery parameters to improve cost-effectiveness and optimize NIR signals. These experiences will provide important information to understand applicability to a variety of sarcoma histologies. Finally, we are interested in developing a multicenter, collaborative trial to better understand how generalizable this approach is to thoracic surgeons at other institutions (please contact author SS if interested). These advances and data, along with additional follow-up, will allow us to better understand how NIR imaging can potentially improve upon the dismal prognosis for patients with metastatic sarcoma.

\section{Webcast}

You can watch a Webcast of this AATS meeting presentation by going to: https://aats.blob.core.windows.net/media/ 18Apr30/25ABC \%202.General \% 20Thoracic $\% 20$ SS/S69\% 20-\%20Part\%201/S69_2_webcast_022220667.mp4.

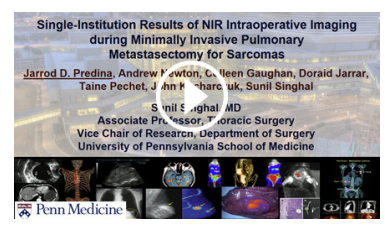

\section{Conflict of Interest Statement}

Authors have nothing to disclose with regard to commercial support.

\section{References}

1. American Cancer Society. Key statistics for soft tissue sarcomas. Available at: https://www.cancer.org/cancer/soft-tissue-sarcoma/about/key-statistics.html. Accessed December 19, 2018.

2. Billingsley KG, Burt ME, Jara E, Ginsberg RJ, Woodruff JM, Leun DH, et al Pulmonary metastases from soft tissue sarcoma: analysis of patterns of diseases and postmetastasis survival. Ann Surg. 1999;229:602-10.

3. Garcia Franco CE, Torre W, Tamura A, Guillen-Grima F, San-Julian M, MartinAlgarra S, et al. Long-term results after resection for bone sarcoma pulmonary metastases. Eur J Cardiothorac Surg. 2010;37:1205-8.

4. Cheang MY, Herle P, Pradhan N, Antippa P. Video-assisted thoracoscopic surgery versus open thoracotomy for pulmonary metastasectomy: a systematic review. ANZ J Surg. 2015;85:408-13.

5. Chudgar NP, Brennan MF, Munhoz RR, Bucciarelli PR, Tan KS, D’Angelo SP, et al. Pulmonary metastasectomy with therapeutic intent for soft-tissue sarcoma. J Thorac Cardiovasc Surg. 2017;154:319-30.e311.

6. Predina JD, Puc MM, Bergey MR, Sonnad SS, Kucharczuk JC, Staddon A, et al. Improved survival after pulmonary metastasectomy for soft tissue sarcoma. $J$ Thorac Oncol. 2011;6:913-9.

7. Cerfolio RJ, Bryant AS, McCarty TP, Minnich DJ. A prospective study to determine the incidence of non-imaged malignant pulmonary nodules in patients who undergo metastasectomy by thoracotomy with lung palpation. Ann Thorac Surg. 2011;91:1696-700.

8. Cerfolio RJ, McCarty T, Bryant AS. Non-imaged pulmonary nodules discovered during thoracotomy for metastasectomy by lung palpation. Eur J Cardiothorac Surg. 2009;35:786-91.

9. Ellis MC, Hessman CJ, Weerasinghe R, Schipper PH, Vetto JT. Comparison of pulmonary nodule detection rates between preoperative CT imaging and intraoperative lung palpation. Am J Surg. 2011;201:619-22.

10. Okusanya OT, Holt D, Heitjan D, Deshpande C, Venegas O, Jiang J, et al. Intraoperative near-infrared imaging can identify pulmonary nodules. Ann Thorac Surg. 2014;98:1223-30

11. Predina JD, Newton A, Lee MK, Singhal S. Near-infrared intraoperative imaging can successfully identify of malignant pleural mesothelioma after neoadjuvant chemotherapy. Mol Imaging. 2017;16:1536012117723785.

12. Predina JD, Newton AD, Desphande C, Singhal S. Near-infrared intraoperative imaging during resection of an anterior mediastinal soft tissue sarcoma. Mol Clin Oncol. 2018;8:86-8

13. Keating J, Newton A, Venegas O, Nims S, Zeh R, Predina J, et al. Near-infrared intraoperative molecular imaging can locate metastases to the lung. Ann Thorac Surg. 2017;103:390-8.

14. Lee JY, Thawani JP, Pierce J, Zeh R, Martinez-Lage M, Chanin M, et al. Intraoperative near-infrared optical imaging can localize gadolinium-enhancing gliomas during surgery. Neurosurgery. 2016;79:856-71. 
15. Schweiger T, Schwarz S, Traxler D, Dodier P, Aigner C, Lang G, et al. Bronchoscopic indocyanine green fluorescence imaging of the anastomotic perfusion after tracheal surgery. Ann Thorac Surg. 2016;101:1943-9.

16. Munabi NC, Olorunnipa OB, Goltsman D, Rohde CH, Ascherman JA. The ability of intra-operative perfusion mapping with laser-assisted indocyanine green angiography to predict mastectomy flap necrosis in breast reconstruction: a prospective trial. J Plast Reconstr Aesthet Surg. 2014;67:449-55.

17. Terasaki H, Inoue $\mathrm{Y}$, Sugano $\mathrm{N}$, Jibiki $\mathrm{M}$, Kudo $\mathrm{T}$, Lepantalo $\mathrm{M}$, et al. A quantitative method for evaluating local perfusion using indocyanine green fluorescence imaging. Ann Vasc Surg. 2013;27:1154-61.

18. Jiang JX, Keating JJ, Jesus EM, Judy RP, Madajewski B, Venegas O, et al. Optimization of the enhanced permeability and retention effect for near-infrared imaging of solid tumors with indocyanine green. Am J Nucl Med Mol Imaging. 2015;5:390-400.

19. Predina JD, Newton AD, Keating J, Barbosa EM Jr, Okusanya O, Xia L, et al. Intraoperative molecular imaging combined with positron emission tomography improves surgical management of peripheral malignant pulmonary nodules. Ann Surg. 2017;266:479-88.

Key Words: sarcoma, metastasectomy, intraoperative imaging, optical imaging, ICG

\section{Discussion}

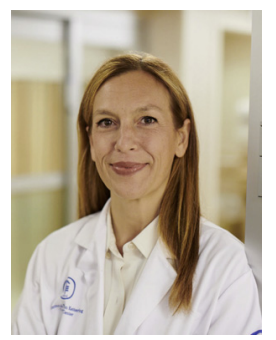

Dr Daniela Molena (New York, NY). I want to congratulate you for the excellent presentation and for being an example for all of us on how you can use technology to improve patient care. You and your colleagues have extensive experience in using intraoperative molecular imaging both with receptor and no receptor targeted dyes, and we saw a beautiful presentation in our skill sessions on Saturday as well. You have used tumor fluorescence for localization but also for margin assessment, and now, as we saw in this presentation, for finding metastasis.

As you have highlighted, among the major limitations of your technique is depth of penetration, and most of these very superficial nodules can potentially be felt by palpation. Is there any way that you think in the future this limitation can be overcome? How deep can you see the fluorescence through the lung and what are your limits?

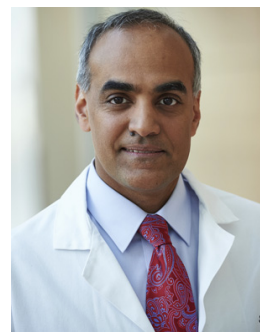

Dr Sunil Singhal (Philadelphia, Pa). Thank you for the question. The key here is really the size of the nodule; the bigger the nodule, the deeper we can see. The other factor relates to the cellularity and grade of the nodule. Higher-grade nodules are more likely to take up the dye.

Dr Molena. When you showed your data on Saturday, you showed different types of receptor-binding dyes that you can use. In this case you didn't use any molecular target at all, just dye alone. So how do you decide what to use before you are going to do a case to find a nodule?
Dr Singhal. Our preference is to use a targeted dye because they are brighter, accumulate 3-fold more, and less nonspecific binding. The problem with sarcomas is there is no single marker, so we use this generic approach, looking more at the macromolecule characteristics such as $\mathrm{pH}$ and angiogenesis. This has been our approach for cancers that don't have a single tumor marker.

Dr Molena. Do you use this for margin assessment or can you use this for margin assessment as well even if it is not molecular targeted?

Dr Singhal. Yes. Among the most valuable features of this intraoperative molecular imaging is imaging on the back table, immediately after taking the specimen out of the patient. You can see where the staple line is and you can determine whether the margins are close.

Dr Molena. Can this dye can be used for any type of metastasis? Have you used it in colorectal metastasis or other types of tumors or just for sarcomas?

Dr Singhal. We have been testing this technology for many types of metastases to the lung. We know this approach can be used for colorectal metastases and we have previously published on that. Melanomas do not seem to work well. I believe that is because the black pigment absorbs the light. For renal cell carcinoma, which is another topic we are exploring, we use a targeted dye that is folate specific. However, we are also looking at the Tumor Glow approach discussed today.

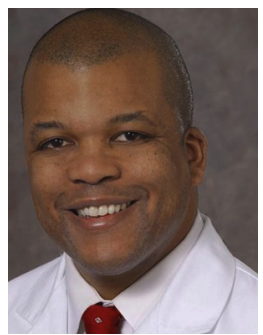

Dr David Tom Cooke (Sacramento, Calif). Wonderful work, as always. I have a question about the logistics of the infusion and the dye. The first question is, How long of an infusion is it? I understand this is part of a clinical trial, so in terms of the practical real world, would this be done in an infusion center or in your outpatient clinic? Do you envision this being covered by insurance payers?

Dr Singhal. We infuse patients over 45 minutes as an outpatient procedure. At first, when we started our work around intraoperative molecular imaging, we were nervous about giving such a high dose of indocyanine green, so we used to premedicate the patients with diphenhydramine. However, the patients could not drive home afterward after taking diphenhydramine. Therefore, we stopped premedicating altogether. We have had no problems, and we have used this approach in more than 800 patients if you include our experience with lung cancer, thymomas, and mesothelioma.

In terms of the infusion, we initially used to do it in the oncology infusion center. Now, we have started a center for precision surgery at Penn and there are 14 surgeons in our group. We image about 200 patients a year who have various tumor types, so we have our own research nurse. That allows us to control our time better. 
In terms of the cost, I think eventually if this reaches Food and Drug Administration approval, then this will be covered, but at this time indocyanine green is not reimbursed for this indication.

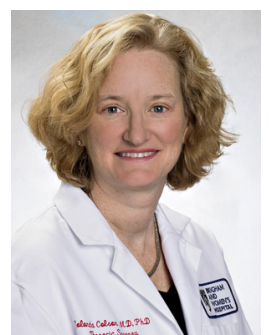

Dr Yolanda L. Colson (Boston, Mass). Thank you very much. It was very nicely done. My question, which you may have already touched on, is the risk that everyone was worried about is the anaphylaxis that was reported when you gave this intravenous injection, and your dose is probably 3 times or more what it normally was. You have done about 800 , and that's where you were giving the diphenhydramine, I assume?

Dr Singhal. Yes. We stopped giving diphenhydramine at around our 200th patient.

Dr Colson. Have all of those 800 patients received this higher dose and have done okay?

Dr Singhal. Yes.

Dr Colson. That's great, because that is the concern with it, that's what everyone was worried about.

Dr Singhal. When I present these data, that is the most common concern. However, we have had no issues and now perform intraoperative molecular imaging anywhere from 6 to 8 times a week.
Dr Colson. Right, exactly.

Dr Singhal. By the way, the key is to infuse over 45 minutes. You can't give this as a push.

Dr Colson. Correct.

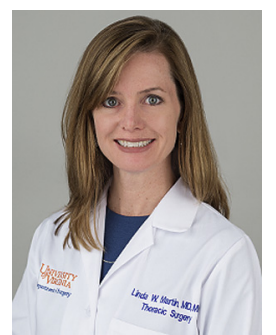

Dr Linda W. Martin (Charlottesville, $\mathrm{Va}$ ). I really enjoyed your talk and actually I think it would be great to explore doing this as a trial, through the Alliance or some other mechanism. I'm surprised how many nodules you found that weren't previously suspected and I was wondering what your protocol was for imaging before surgery, and if you have guidelines about how thin the computed tomography scan cuts should be.

Dr Singhal. All patients get a 1-mm computed tomography scan. Once I see a patient, we typically go to the operating room within 2 to 3 weeks; it is not a prolonged interval. The problem we have in this trial, or in any center, is no single center has enough patients undergoing pulmonary metastectomy to do a large-scale trial. We see maybe 10 to 15 metastatic sarcoma patients a year. I would be very enthusiastic for the opportunity to collaborate with other institutions so we could conduct a larger trial with a large number of patients. 